\title{
Quiet-day ionospheric currents and their application to upper mantle conductivity in Australia
}

\author{
W. H. Campbel1 ${ }^{1}$, C. E. Barton ${ }^{2}$, F. H. Chamalaun ${ }^{3}$, and W. Welsh ${ }^{2}$ \\ ${ }^{1}$ World Data Center A, NGDC/NOAA, 325 Broadway, Boulder, CO 80303, U.S.A. \\ ${ }^{2}$ Australian Geological Survey Organisation, GPO Box 378, Canberra, ACT 2601, Australia \\ ${ }^{3}$ School of Earth Sciences, Flinders University of South Australia, GPO Box 2100, Adelaide, SA 5001, Australia
}

(Received December 10, 1996; Revised September 25, 1997; Accepted December 10, 1997)

This study concerns the use of selected geomagnetic field records to establish the 1990 quiet-day current system $(S q)$ for Australia and to use the ionospheric current source of $S q$ for a determination of the Earth's deep electrical conductivity. The primary data set came from a chain of eight, three-component magnetometer stations that was operated along a north-south line in central Australia. Additional records, necessary for boundary conditions, were added to the data set. A regional spherical harmonic analysis (SHA) allowed the separation of the internal and external field contributions to the $S q$ variations. Mapping of the equivalent ionospheric current from the external field showed that the $S q$ contour focus passed near the $-30^{\circ}$ geomagnetic latitude of central Australia with a $5^{\circ}$ latitude variation between winter and summer and a corresponding change from about 80 to $200 \mathrm{kA}$ in strength. A special transfer function allowed the computation of an equivalent conductivity-depth profile of central Australia from the paired external and internal coefficients of the SHA. A regression line through the conductivity estimates gives a profile that starts at $0.025 \mathrm{~S} / \mathrm{m}$ for a depth of $130 \mathrm{~km}$, rising gradually to about $0.045 \mathrm{~S} / \mathrm{m}$ at $250 \mathrm{~km}$, then steepens to $0.11 \mathrm{~S} / \mathrm{m}$ at $360 \mathrm{~km}$ and rises moderately to $0.13 \mathrm{~S} / \mathrm{m}$ at $470 \mathrm{~km}$ near the base of the upper mantle. No data were obtained through the mantle transition zone. Computations gave $0.18 \mathrm{~S} / \mathrm{m}$ in the region of $800 \mathrm{~km}$ depth. Previous conductivity models for the upper mantle beneath central Australia, although less specific in values, are consistent with our profile. At depths greater than $500 \mathrm{~km}$, the regression profile is in agreement with the conductivity distribution beneath the Tasman Sea determined from seafloor magnetotellurics, although both measurements lack high resolution at such depths.

\section{Introduction}

A fluctuating electric current flowing in the Earth's atmosphere causes corresponding electric currents to flow in the conducting Earth below the source current. The magnitude, direction, and depth of penetration of the induced currents are determined by the characteristics of the source currents as well as the distribution of electrically conducting materials in the Earth. At the Earth's surface observatories, magnetometers measure the composite of external (source) and internal (induced) field components from the currents. This paper concerns the quiet-day field variations, their separation into external and internal contributions, and the use of this separation not only to establish the ionospheric current system but also to profile the electrical conductivity of the deep Earth.

Through the entire year of 1990 the Australian Geological Survey Organisation operated a chain of geomagnetic observatories near a longitude line through central Australia from Darwin to Adelaide. Data from these stations provided the opportunity to study the ionospheric quiet-day current system and to use this system in determining the Earth's upper mantle electrical conductivity. Below we will give the brief background to show how such currents and conductiv-

Copy right (C) The Society of Geomagnetism and Earth, Planetary and Space Sciences (SGEPSS); The Seismological Society of Japan; The Volcanological Society of Japan; The Geodetic Society of Japan; The Japanese Society for Planetary Sciences. ity were obtained from the chain of magnetic records.

By the late nineteenth century scientists had established laws governing electric and magnetic phenomena. Maxwell (1873) had devised the short list of differential equations that brought together all these laws. Given a set of field measurements, Maxwell's formulation allowed the computation of possible causative current configurations. When the source current can be properly represented by an additive series of elemental currents, then each elemental current can be treated separately as behaving Maxwell's equations; such decomposition is important to our analysis method.

For the situation in which field measurements are available about a spherical surface that separates the source from the induced currents (and a current doesn't flow across this surface), Gauss (1838) devised a special solution of the differential electromagnetic field equations that is separable in the spherical coordinates $r, \theta$, and $\phi$. In Gauss's solution, the field terms that represent radial dependence appear as two series-one with increasing powers of the sphere radius, $r$, and one with increasing powers of $1 / r$. As the value of $r$ becomes larger (outward from the sphere) the first series produces an increased field strength, as if approaching external current sources. As the value of $r$ decreases (toward the sphere center) the second series of $1 / r$ terms indicate increased field strength, as if approaching internal current sources. Gauss had devised the way to separately represent the currents that were external and internal to his analysis 
sphere. For these external and internal series, there are individual spherical harmonic analysis (SHA) polynomial (Legendre) terms, each having two indices, degree $m$ and order $n$. The full field is then represented as paired (external and internal Legendre terms) elemental parts, each satisfying the physical laws.

Gauss applied the SHA method to the global field observations and verified that most of the Earth's main field originated from internal sources. By the turn of the century, the method was also used to show that the daily, quiet-time geomagnetic field variations came mostly from sources of current external to the Earth (Schuster, 1889, 1908); this finding led to the discovery of the ionosphere. We will analyze the quiet fields with this SHA to separate the $S q$ ionospheric source currents from the induced currents within the Earth.

Chapman (1919, and Chapman and Bartels, 1940) introduced a novel variation of the analysis for the quiet day fields. He treated a longitude line of observatories as riding with the Earth under a fixed, subsolar, ionospheric current system. In that way, at each latitude the quiet daily variation $(S q)$ of field in 24 hours represented the field measured around $360^{\circ}$ of a sphere. We can call this "cloning" a sphere from a longitudinal "slice" sample. From an induction point of view, this mathematical representation implies a spherical symmetry of the Earth's conducting properties; but the evaluation is only applicable to the original slice longitude. Matsushita and Maeda (1965) used Chapman's method to complete a major global analysis of external and internal $S q$ for north-south continental sectors of the Earth during the solstitial and equinoctial seasons of the solar active year 1958. Variations of the analytical methods have been compared by Campbell (1990).

In 1970 Schmucker published the transfer equations necessary for obtaining a conductivity versus depth profile from the separated external and internal SHA fields. A subsequent series of publications extended the Schmucker work with conductivity analyses of continental half-sectors (Campbell and Anderssen, 1983; Campbell and Schiffmacher, 1986, 1988a, 1988b; Campbell, 1987, 1997;
Aurora et al., 1995). The method used by these authors first determined a yearly $S q$ change from the selected quiet-day field variation. Then their mathematical technique cloned a sphere (necessary for the Gauss external-internal separation) from the area of interest by modeling opposite hemisphere variations from 6-month, time-shifted versions of the principal data set (called the "mirror-slice" method). After performing the SHA and extracting the external and internal parts of the $S q$ twice each month through the year, each pair of $n$ and $m$ indices, in each of the 24 analyses, represented the contribution from an elemental field for which the transfer function could provide a single value of corresponding conductivity and depth. A locally weighted regression fitting of the combined results produced a robust conductivity profile of the upper mantle beneath the study area.

A recent investigation of the external source currents and deep electrical conductivity for the India-Siberia region (Campbell et al., 1992, 1993; Arora et al., 1995) is the latest example of this analytical method. Because the analysis technique, mathematical formulation, and computer algorithms of these authors has been followed exactly (except for adjustments to the Australia region) in the study reported now, it is not necessary to repeat those equations here. However, a general description of the methodology is provided in Sections 3 and 4 below; the interested reader can refer to the India-Siberia papers for greater details.

\section{The Magnetic Observations}

Eight magnetometers had been deployed along a northsouth transect across Australia to record magnetic field variations during a 13-month interval commencing in November 1989. The program was part of an "Australia-Wide Array of Geomagnetic Stations" (AWAGS) experiment to investigate the space-time patterns of the geomagnetic field over the entire continent and to determine the inductive response of the crust and ocean margins (Chamalaun and Barton, 1993a, b). The instruments were developed at Flinders University of South Australia as self-contained fluxgate units with solid-state data logging, a sensitivity of about 1 $\mathrm{nT}$, and capacity for operating unattended for 4 months

Table 1. Data source locations.

\begin{tabular}{|c|c|c|c|c|c|}
\hline \multirow[t]{2}{*}{ Name } & \multirow[t]{2}{*}{ Code } & \multicolumn{2}{|c|}{ Geographic } & \multicolumn{2}{|c|}{ Geomagnetic } \\
\hline & & lat $\left({ }^{\circ} \mathrm{N}\right)$ & lon $\left({ }^{\circ} \mathrm{E}\right)$ & lat $\left({ }^{\circ} \mathrm{N}\right)$ & lon $\left({ }^{\circ} \mathrm{E}\right)$ \\
\hline Equatorial Region & EQX & 9.77 & 134.42 & 0.00 & 205.0 \\
\hline Low Latitude & LOX & 1.79 & 133.78 & -8.00 & 205.0 \\
\hline Port Moresby & PMG & -9.41 & 147.15 & -17.80 & 220.0 \\
\hline Darwin & DAR & -12.42 & 130.87 & -22.40 & 203.4 \\
\hline Daly Waters & DYW & -16.27 & 133.37 & -26.08 & 206.4 \\
\hline Tennant Creek & TCK & -19.63 & 134.18 & -29.35 & 207.6 \\
\hline Alice Springs & ASP & -23.81 & 133.90 & -33.54 & 207.7 \\
\hline Mount Dare & MTD & -26.50 & 135.25 & -36.09 & 209.5 \\
\hline Etadunna & ETA & -28.72 & 138.63 & -37.96 & 213.6 \\
\hline Mable Creek & MAC & -28.95 & 134.32 & -38.62 & 208.8 \\
\hline Composite ETA/MAC & CEM & -28.80 & 136.50 & -38.26 & 211.2 \\
\hline Port Augusta & PTA & -32.48 & 137.75 & -41.79 & 213.2 \\
\hline Macquarie Island & MCQ & -54.50 & 158.95 & -60.42 & 244.5 \\
\hline High Latitude & HIX & -67.00 & 135.00 & -75.94 & 225.2 \\
\hline South Pole Region & SMX & -80.00 & 130.00 & -86.10 & 292.8 \\
\hline
\end{tabular}




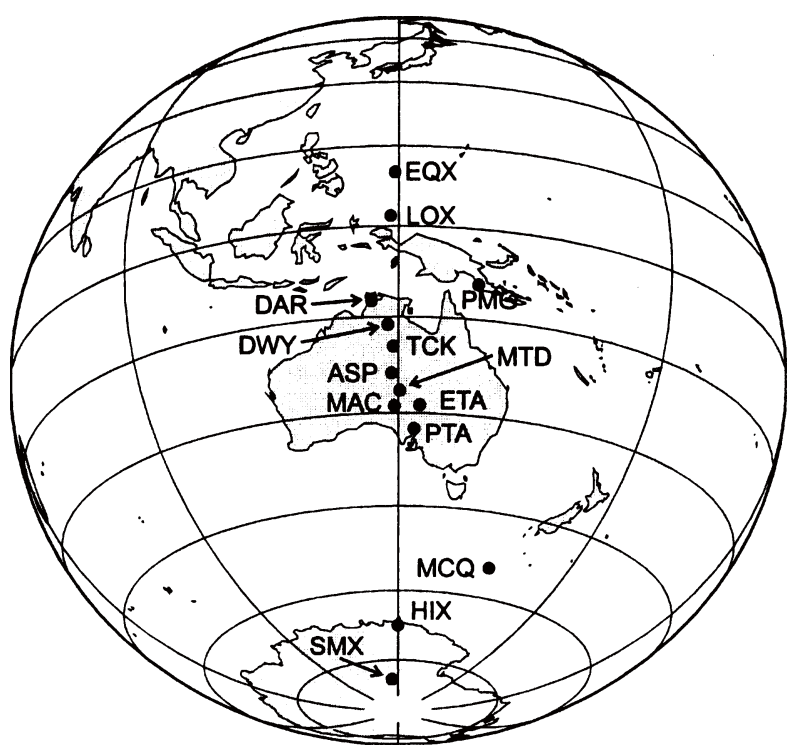

Fig. 1. Location of sites used in the analysis: eight AWAGS stations within Australia, two magnetic observatories adjacent to the Australian continent (PMG and MCQ), and four boundary locations (EQX, LOX, HIX, and SMX) for which fields were reconstructed using the global $S q$ model. All locations are specified in Table 1.

(Chamalaun and Walker, 1982). Three components of the field, together with temperature and time, were recorded at one-minute intervals. For our analysis, 10 data values (centered about each 10-min marker) were averaged to provide 10-minute field samples.

Our analysis technique requires a determination of the $S q$ quiet-day fields along a full longitude line from the equator to the pole in one hemisphere. It has been shown that the principal contribution to the potential function defining $S q$ comes from fields weighted with respect to the proximity of the $S q$ current vortex center (Arora et al., 1995). That focus location occurs near $-30^{\circ}$ geomagnetic latitude over central Australia (Lilley, 1975; Parkinson, 1977; Campbell and Schiffmacher, 1987; Stening and Hopgood, 1991). Therefore, those AWAGS stations nearer latitudes of central Australia would carry the major weight in defining the conductivity profile; stations near the equator and pole provide only the necessary boundary conditions to fill-in a hemispherical profile. Accordingly, to augment the Australian station set, concurrent data from nearby Port Moresby (PMG) to the north and MacQuarie Island (MCQ), to the south, were added. Then, to establish the necessary boundary conditions near the equator and the pole, field values were computed at four locations in these bordering regions (EQX, LOX, HIX, and SMX) from the standard quiet-day field model WDCA-SQ1 (Campbell et al., 1989; available as program SQ1MODEL.EXE on world wide web at http:// www.ngdc.noaa.gov/seg/potfld/utilwhc.html).

Table 1 is a list of the station codes, geographic locations, and geomagnetic coordinates of the data sources for our analysis. Figure 1 is a map showing the locations for the data sets. The two adjacent AWAGS stations, Etadunna (ETA) and Mable Creek (MAC) were occupied at complementary half-years. During the analysis, after rearrangement into local time, data from these two stations were concatenated, given a new composite name (CEM), and taken to be located at the mean position of the original stations.

\section{The Quiet-Day Field}

On days undisturbed by solar-terrestrial field and particle activity the geomagnetic records from a surface observatory display a smooth variation of field during the daylight hours. These variations are dominated by 24-, 12-, 8-, and 6-hr spectral components that change slowly from day to day through the seasons. Laboratory and in-situ studies have demonstrated that these variations arise from dynamo currents created when the conducting $E$ region and lower $F$ region of the ionosphere are moved through the main field of the Earth by tidal and thermospheric winds (see Chap. 2 in Campbell, 1997).

Figure 2 illustrates the typical summer daytime magnetograms for geomagnetic northward $(H)$ and eastward $(D)$ components of field variations at the Earth's surface between $50^{\circ}$ north and south geomagnetic latitudes. The variations follow a changing pattern that can be separated into local time (LT) pre-noon (0600 to $1200 \mathrm{LT}$ ) and afternoon (1200 to $1800 \mathrm{LT}$ ) periods; vector arrows in the right column indicate the dominant field direction at each period. The wire segment sketch in the column marked "current" illustrates the equivalent overhead current configuration that would give rise to the dominant morning and afternoon local-time field directions shown as vectors. The vortex pattern at the bottom of the figure combines the current elements to illustrate how a system of two oppositely directed current vortices in the daytime ionosphere produce the observed quiet-day fields at the Earth's surface.

Figure 3 illustrates how, in 24 hours, a southern longitude chain of magnetic observatories on the Earth's surface, will pass under an ionospheric dynamo current vortex. These locations will sample the Southern Hemisphere of a spherical surface that separates the external (source) and internal (induced) fields on a quiet day. In the analysis, small differences in longitude of the stations are adjusted by arrangement of the field variations in local time. An analysis field pattern for comparable locations in the opposite hemisphere is obtained by shifting the date in 1990 by 6 months and changing signs of the appropriate field components to conform to a reversed source-current vortex (Campbell, 1990).

The analytical method can be visualized in this way. Consider the situation that we wish to explore the currents and conductivity for an Earth half-sector (as a half-section of an orange). We need a full sphere to use Gauss's method of separating the external and internal currents, and need the SHA coefficients for the transfer function defining the conductivity profile. We clone our hemisphere by the transformation of 24 hours in time to $360^{\circ}$ degrees in longitude (Campbell, 1990). This procedure is equivalent to saying that for the mathematical analysis, deep conducting layers uniformly similar (in longitude) to that below the stations is modeled. That is as if we have cloned a halforange from our half-section. Next, we mirror values to the opposite analysis hemisphere by simply shifting the variation at comparable locations by 6 months (summer in one hemisphere matches winter in the other) and changing some 

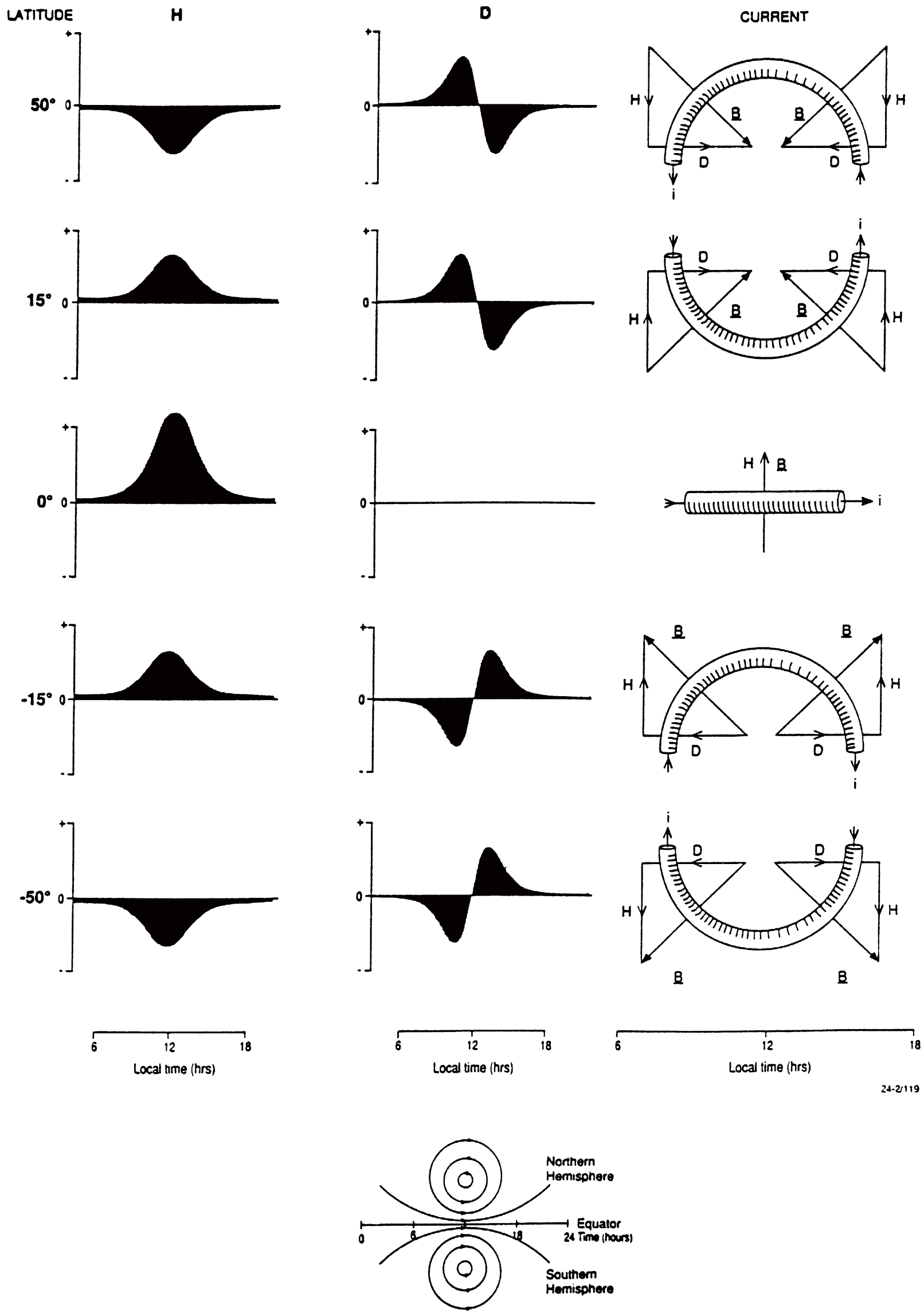

Fig. 2. $S q$ current vortex patterns inferred from $H$ and $D$ field directions. Equivalent current that would flow in above-surface wire segments to cause the observed $H$ and $D$ fields are sketched to the right of each $H$ and $D$ diagram along with the $H$ and $D$ field vectors. The daytime fields in the Northern and Southern Hemispheres are consistent with the $S q$ ionospheric current vortices depicted at the bottom of the figure. 


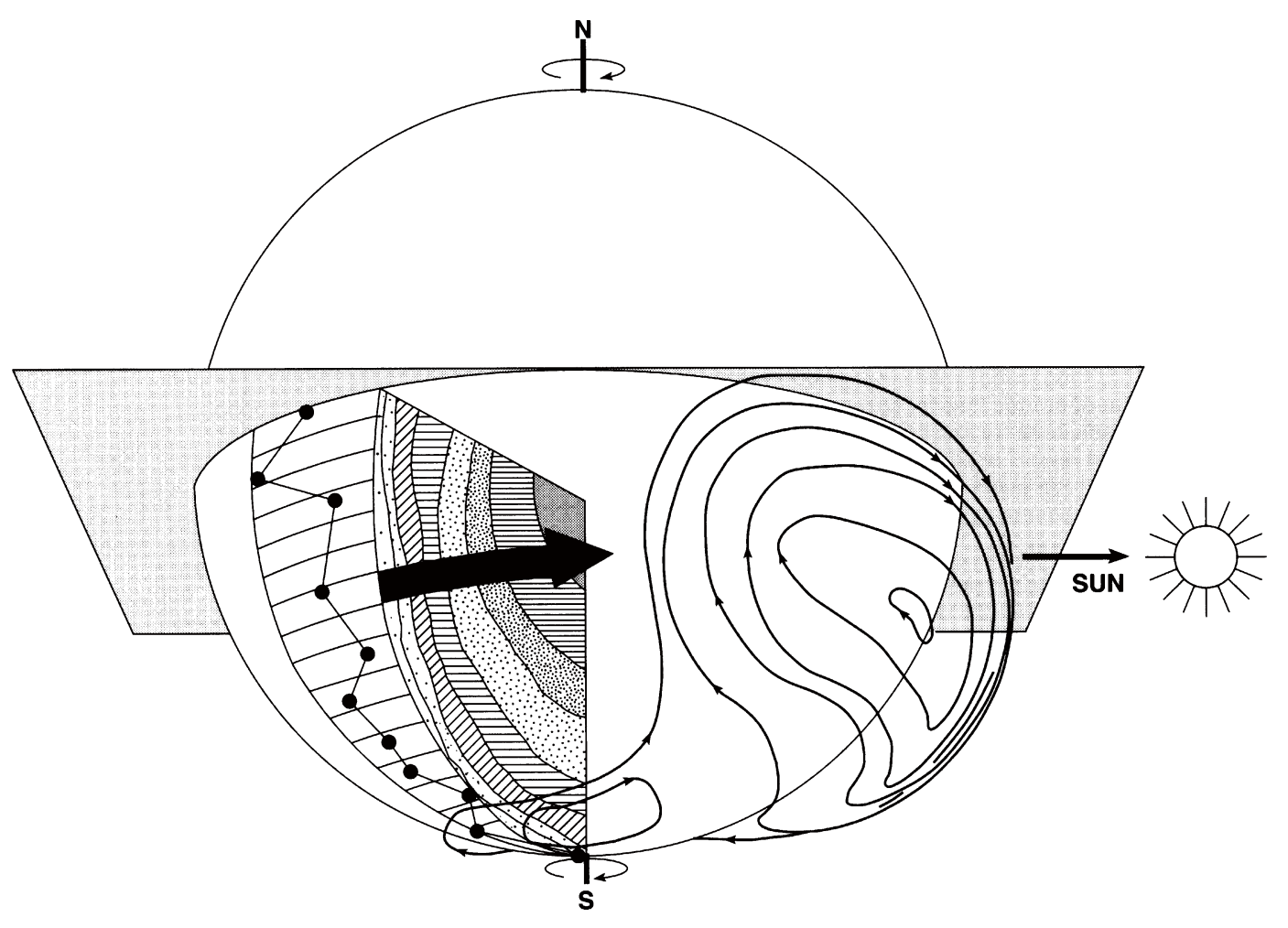

Fig. 3. Procedure for mapping the $S q$ equivalent current system (nested curved lines) using a north-south transect of geomagnetic field observatories (dots connected by straight lines) at the Earth's surface. The current system is assumed to be fixed, with respect to the Sun-Earth line, at about 100 $\mathrm{km}$ above the Earth's surface while the observatories rotate beneath the currents. The 24-hour magnetometer records at the line of observing stations represent a sample of the current system over a complete analysis hemisphere.

field signs. Then our sphere is complete (a whole orange from the half-section) and we can proceed with the SHA. However, the analysis results apply only to the initial longitude chain of stations in the region from which the data were obtained. In effect, the cloning and mirroring are boundarycondition devices to allow the mathematical analysis of a limited region of interest. The procedure is analogous to a Fourier analysis, in which a represented variation through a specified time interval is assumed to repeat indefinitely for intervals before and after the principal interval so that a special mathematical procedure can be applied.

Figure 4 is a flow diagram to illustrate the full data processing routine. The relevant equations can be found in the references to the method given in Introduction (Section 1). The analysis starts with three categories of input: the selected quiet days of 1990, the 3-component measurements of field values at the observatories, and the boundary value field variations (near the equator and pole) synthesized from the global quiet day model.

There were 63 days in 1990 in which the global geomagnetic disturbance index, $K p$, had all 8 daily values less than $3_{\mathrm{o}}$ and, at the same time, had no previous day values greater than $4_{o}$ (see p. 160 of Campbell, 1997, for explanation of the $K p$ index). These days were taken as preliminary "quiet day" recordings. We shall see that more severe restrictions later reduce this number. All observatories had 10 -min sample records available except Port Moresby where the scalings provided were $60-\mathrm{min}$ average values. The original recordings of field were in Universal Time (UT) as orthogonal north, east, and into-the-earth components of field as $X, Y$, and $Z$. Considerable effort was expended to eliminate random spikes in the original records and to remove sudden shifts in baseline levels.

After the $X$ and $Y$ records were transformed to the magnetic northward $(H)$ and eastward $(D)$ coordinate system, the data for each component were Fourier analyzed for each quiet day. With a removal of the daily trend and baseline, the spectral coefficients were defined to the sixth harmonic (24, 12-, 8-, 6-, 4.8-, and 4-hr Fourier components). These coefficients were then appropriately phase-shifted to represent the variation in mean local time at each station.

At this point, each daily coefficient was examined for its consistency with gradual change through the year so that disturbed days, not initially indicated by large $K p$ values, could be removed. If a coefficient value varied more than two standard deviations from the four adjacent neighbors (two earlier and two later coefficients), the value was replaced by the mean of the four neighboring coefficients, and the date was recorded in a temporary "BADDAY" list. After all components of every station were evaluated for such outliers, the temporary BADDAY list was examined. If there were 10 or more outliers for a given day, that day was transferred to a permanent BADDAY file. These BADDAY selections were then removed from the selected quiet days. There were 26 days in the BADDAY file; that left 37 days for the $S q$ study. Fortunately, these days were adequately distributed through the year to represent the seasonal change. A determination of coefficient values at exactly evenly spaced intervals through the year was obtained from a linear extrapolation using the selected quiet-day values. 


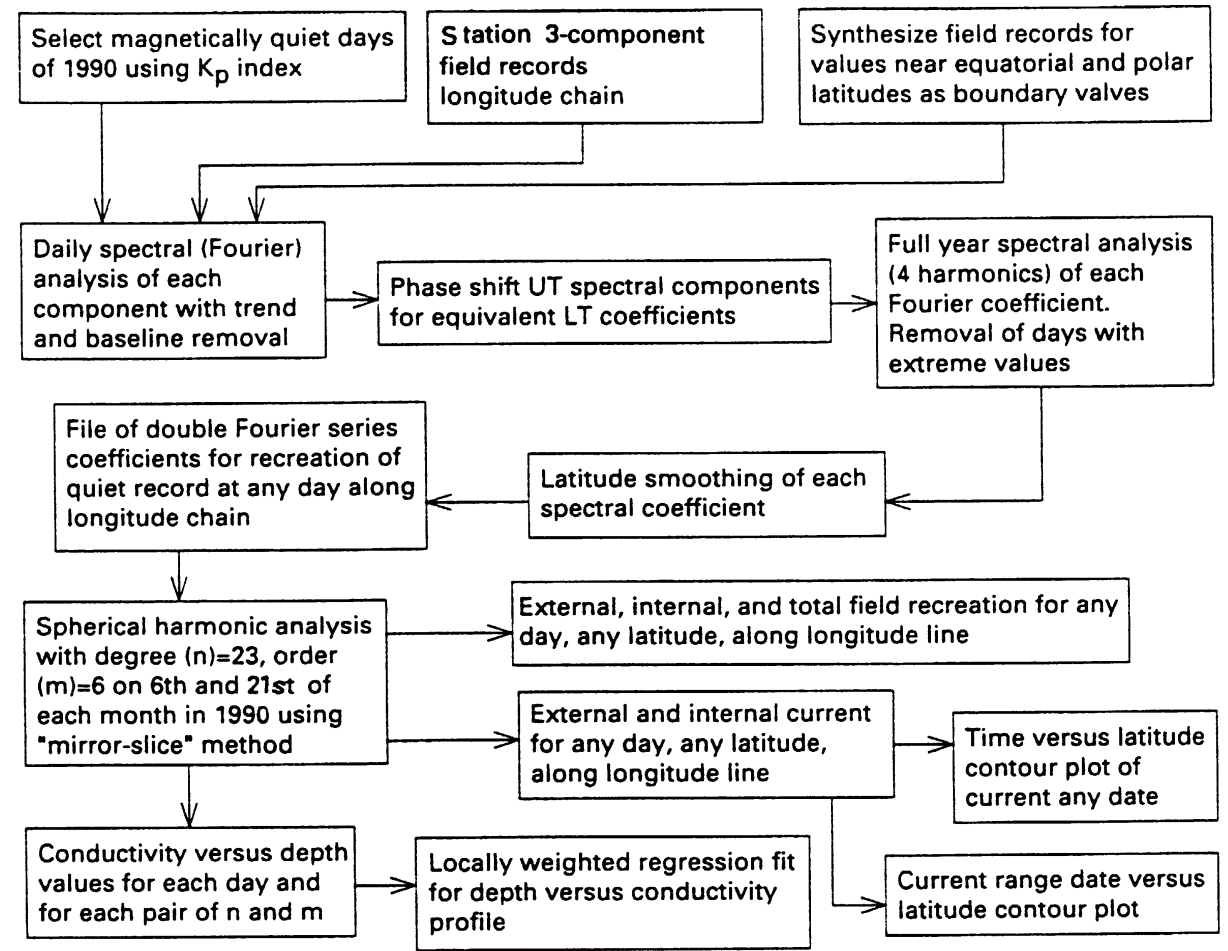

Fig. 4. Flow diagram for analysis of the quiet-day field variations and upper-mantle conductivity. The analysis follows the procedure described by Campbell (1990), Campbell et al. (1992, 1993), and Arora et al. (1995).
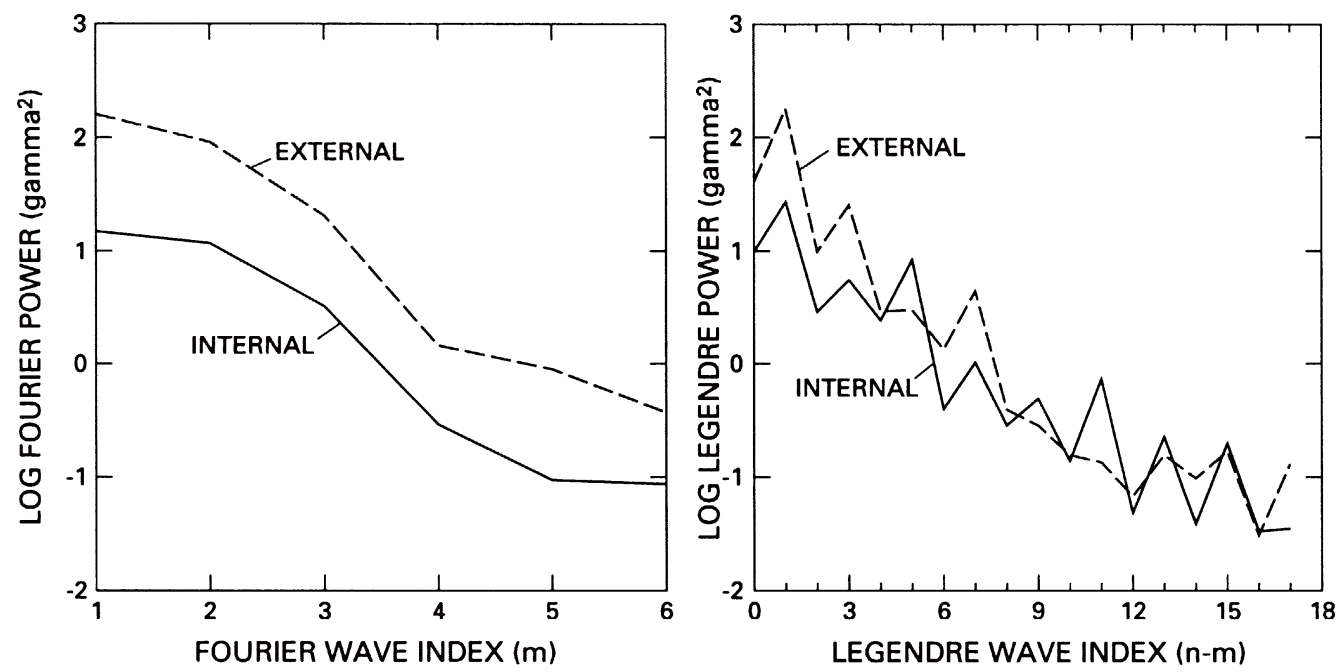

Fig. 5. Spectral power for external and internal parts of the field obtained from the spherical harmonic analysis, SHA (see Campbell, 1990, for the mathematical expressions). Using the SHA degree and order indices, $n$ and $m$, the left diagram shows the daily variation contributions as log Fourier power versus index $m$; right diagram shows the latitude contributions as log Legendre power versus $(n-m)$. A power value of 0.1 corresponds to an amplitude of the spectral component of about 0.3 gamma.

A second, full-year Fourier analysis was carried out on each station data set of the evenly spaced, year's coefficients to determine the mean, linear trend, and the 12-, 6-, 4-, and 3 -month harmonic variations. Only the annual and semiannual variations were of significant size. The final, double Fourier series representation of station coefficients then allow the reconstruction of the $S q$ for the station on any day of the year.

The next task was to model the best smooth latitude representation of the quiet fields. Again, each Fourier coef- ficient was treated separately as a value that had an identified latitude (of the contributing location). The smoothing started with a linear interpolation between the values, and involved polynomial fitting and running-variable-window smoothing (see Campbell et al., 1992) to yield $2^{\circ}$ geomagnetic latitude representations of each coefficient. The latitude file of these smoothed double Fourier series values (called file 90A1-3X8.DAT) allowed us to recreate the three orthogonal field components on any quiet day of 1990 at any selected latitude within the analysis range. 


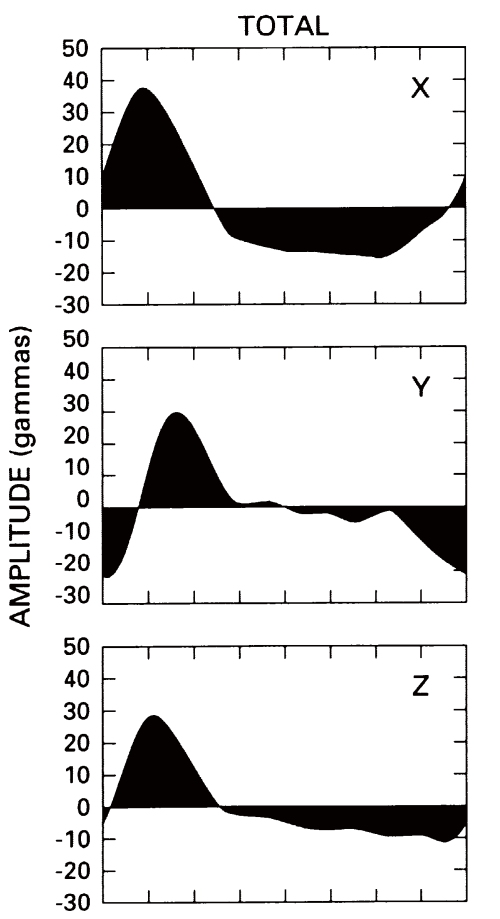

DARWIN, JANUARY
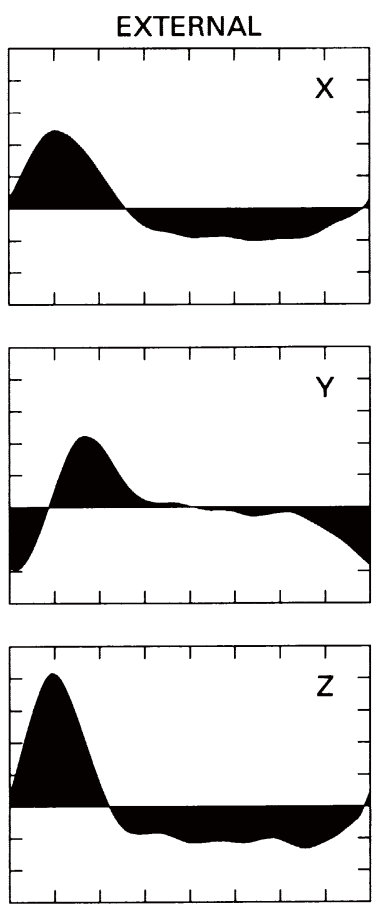

PORT AUGUSTA, JANUARY
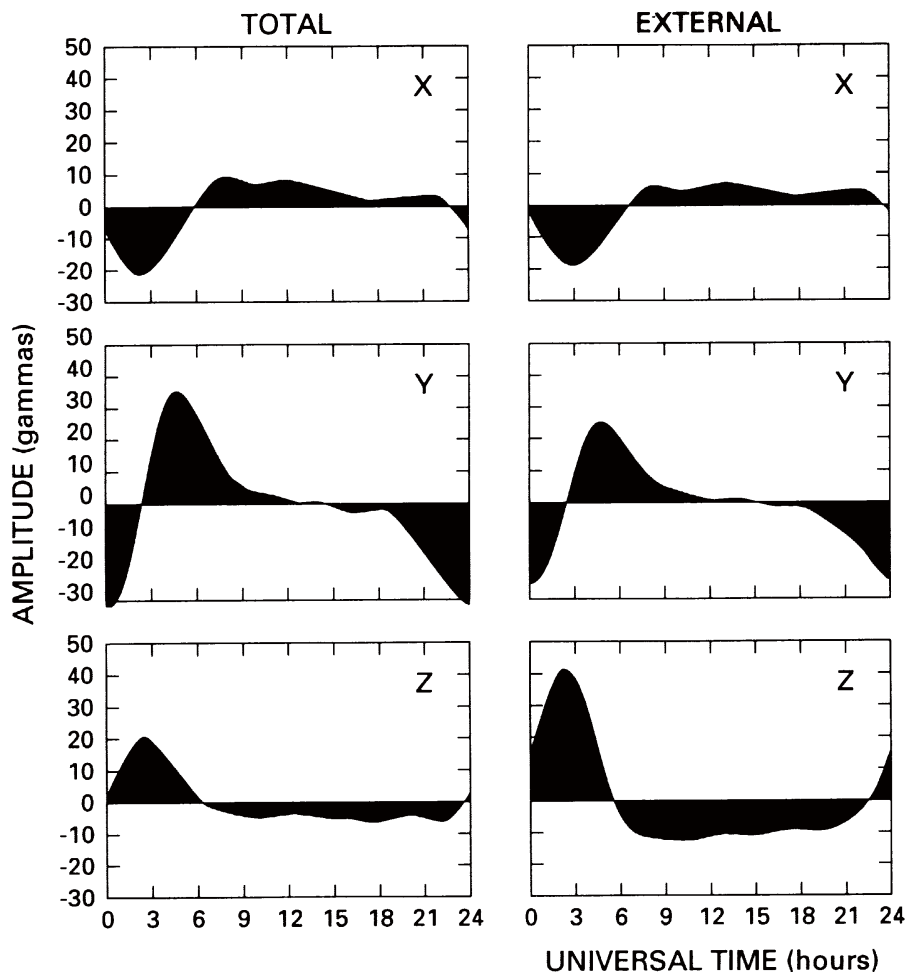
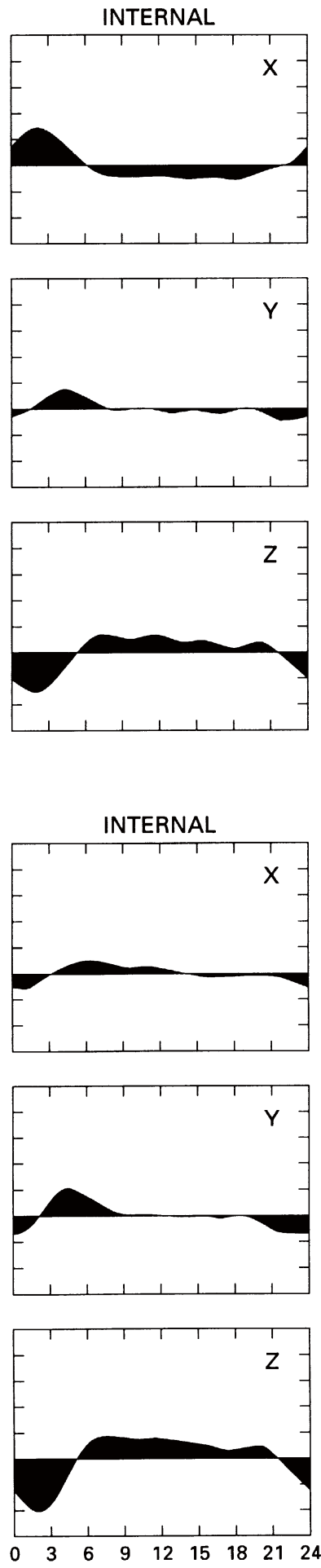

Fig. 6. Sample of the three-component $(X, Y$, and $Z$ ) quiet day field variations for January 15 at the northern and southern ends of the AWAGS station chain, Darwin and Port Augusta. These sites represent locations equatorward and polarward of the central Australia quiet-time current vortex. Amplitudes ( 1 gamma $=1$ nanotesla) are indicated to the left. Universal Time is given in hours on the horizontal axis. Left column represents the total (external plus internal) $S q$ variation; center column displays the external (source) field contribution; right column displays the internal (induced) field contribution. Note the phase and amplitude changes between the last two columns and between the stations.

\section{External and Internal Fields and Currents}

For the 6th and 21st day of each month of 1990 a spherical harmonic analysis (SHA) of field data sets, obtained from the file 90A1-3X8.DAT, was computed to degree $n=23$ and order $m=6$. This means that the maximum number of waves used to represent the field along a $360^{\circ}$ great circle of geomagnetic longitude was 23 ; or $360 / n=15.7^{\circ}$ in wavelength. In the 24-hr representation of the field variation, the shortest component was a $24 / m=4$-hour oscillation.

Figure 5 shows the power distribution of the separated 
external and internal coefficients for the SHA of the summertime (21 December) quiet-day fields. The distribution of Fourier power (for order $m$ wave index) indicates that by $m$ $=6$ the external fields have entered the noise levels near 0.6 gamma $\left(\log\right.$ of 0.36 gamma $\left.^{2}=-0.44\right)$. We should expect this result because the quiet days have only small contributions below $m=4$ (the 6 -hr component). Figure 5 also shows that the external Legendre power reaches this same gamma level at $n-m$ values between 8 and 9 . That corresponds to wavelengths of about $360 /(n-m+1)=36^{\circ}$ to $40^{\circ}$ along a great circle of longitude; variations of shorter wavelengths are near noise levels. Some randomness of behavior seen in the figure reflects residual data noise.

The external and internal spherical harmonic coefficients allow us to separate the corresponding contributions to the surface field. In Fig. 6 we show the reconstructed $X, Y$, and $Z$ components of fields for representative winter (21 June) and summer (06 January) conditions at the northern (Darwin) and southern (Port Augusta) ends of the chain of
AWAGS stations. Here, the $H$ and $D$ components have been rotated to the $X$ and $Y$ directions and translated from Local Time to Universal Time to aid the Australian workers in comparing these results with their typical recordings. Note the relative amplitudes and phase differences of the external and internal contributions. These features arise from the effects of the conducting Earth.

The mapping of external (source) equivalent currents above the study region is readily established from the output of the SHA analysis (Campbell and Schiffmacher, 1987). Displaying the $21 \mathrm{st}$ of each month, Fig. 7 illustrates that annual pattern of current contours forming the typical clockwise Southern Hemisphere $S q$ vortex. There is $10 \mathrm{kA}$ of current flowing between each contour line in the direction indicated by the arrows. A polar cap midnight zero level is assumed for the plot. Note the focus summertime (January) maximum of about $200 \mathrm{kA}$ and the wintertime (June) minimum of about $80 \mathrm{kA}$. Code letters for the sites (Table 1) which were sources of the magnetic data are indicated to the

1990 AWAGS QUIET CURRENTS (10 kA)
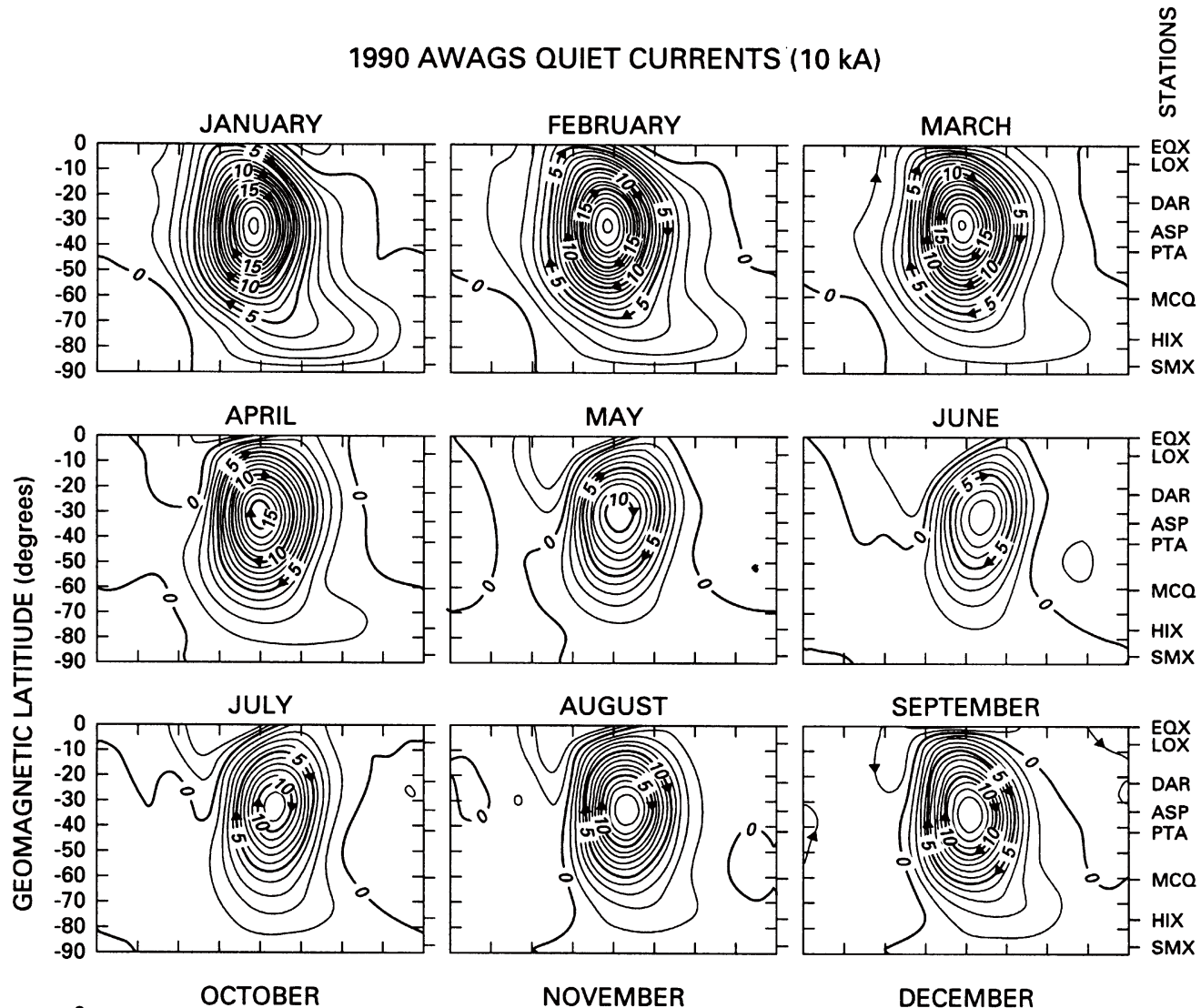

AUGUST
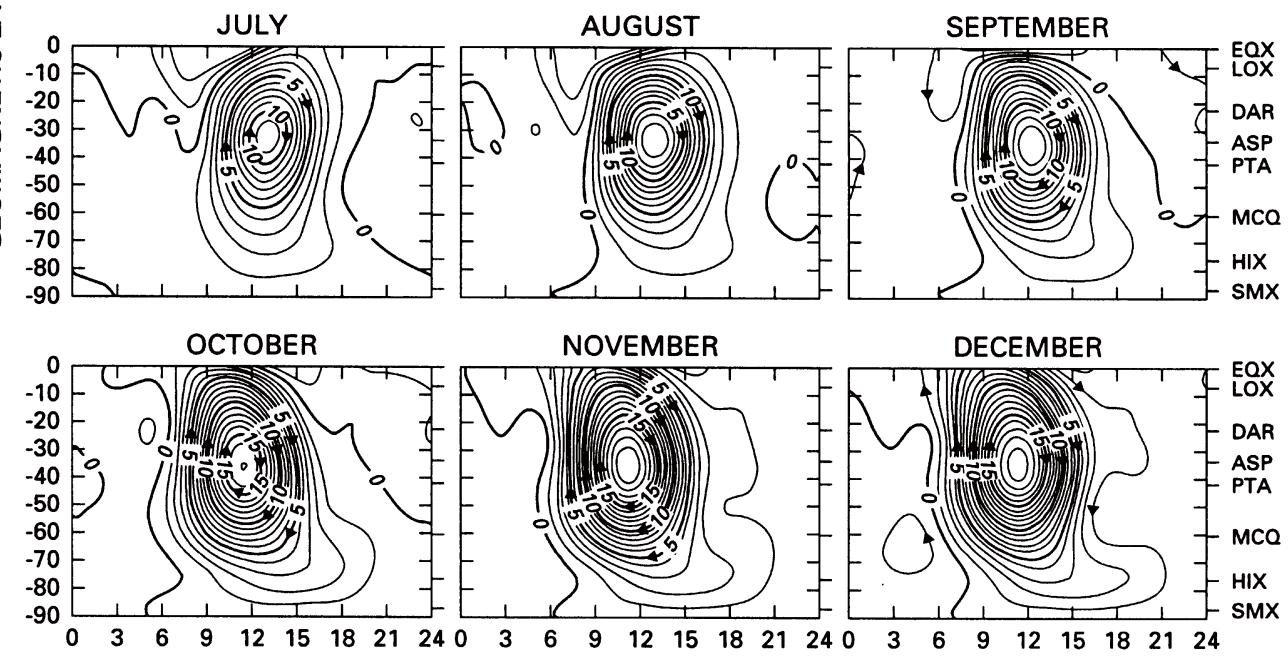

LOCAL TIME (hours)

Fig. 7. The annual pattern of equivalent external current contours above the study region in central Australia computed from the SHA for the 15 th of each month in 1990. Current values are indicated in $10 \mathrm{kA}$ units; there is $1 \mathrm{kA}$ of current flowing between pairs of contour lines (more current in dense contour regions). The typical Southern Hemisphere clockwise vortex is displayed with changing focus positions illustrated in Fig. 8. 


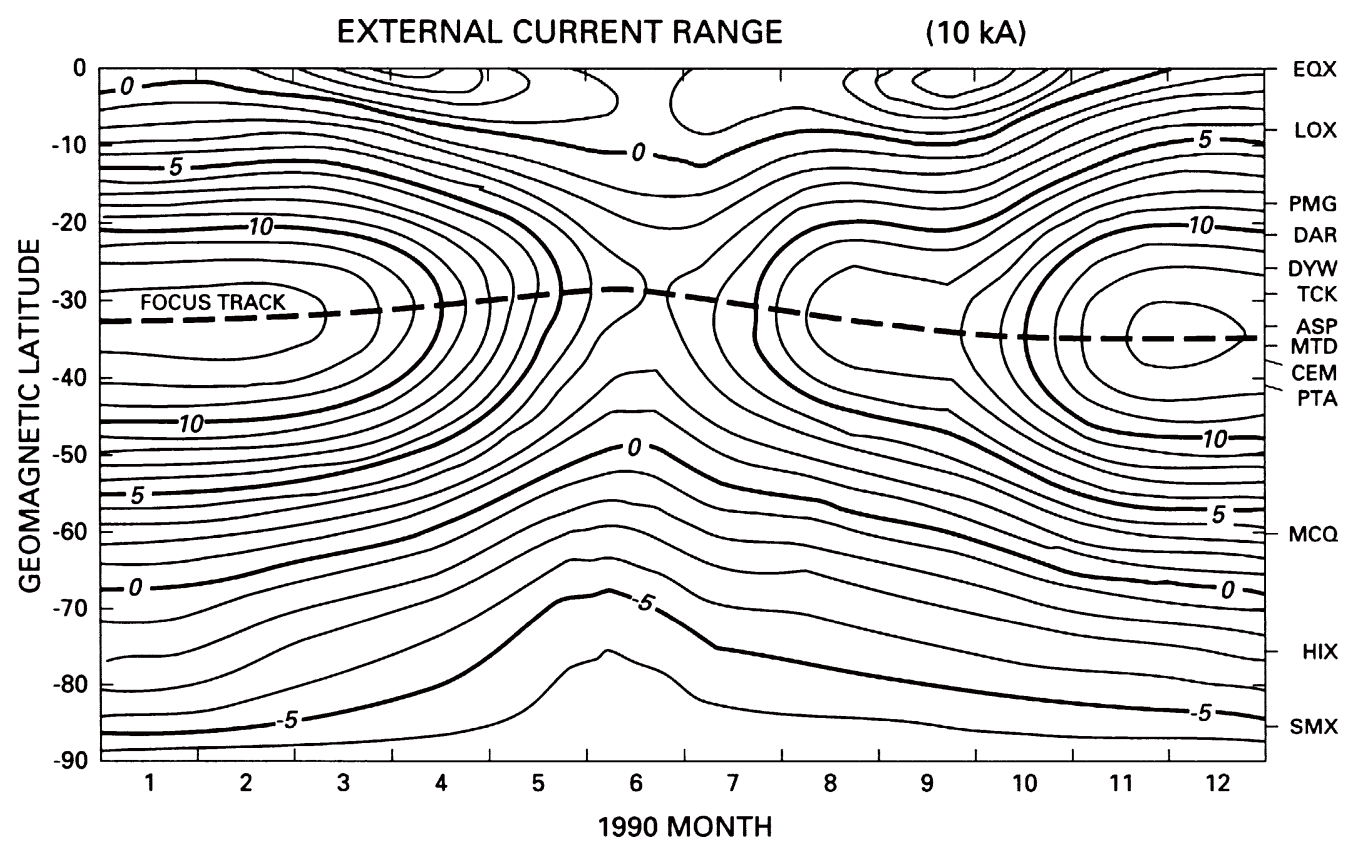

Fig. 8. Day of year (months in 1990 indicated at bottom) versus geomagnetic latitude (given at left) representation of the daily external current range, at each latitude, for the $S q$ equivalent currents displayed in Fig. 7. Contour units are $10 \mathrm{kA}$. To the right is the latitude location of the analysis stations, indicated by the code letters of Table 1. The seasonal track of the $S q$ vortex focus (represented by the dashed line) is determined from the latitude of maximum range for each day.

right. Note the $S q$ focus location near Alice Springs (ASP).

Figure 8 summarizes seasonal features of the monthly external current contours. Here, a single parameter, the daily range of external current, is shown for the analyzed 6th and 21 st of each month through the spread of southern latitudes followed in the SHA. The contours are given in $10 \mathrm{kA}$ steps. The track of the daily $S q$ vortex focus near $-30^{\circ}$ geomagnetic latitude is indicated by a dashed line. In 1990 there was about $5^{\circ}$ northward movement of that focus from summer to winter.

\section{Upper Mantle Conductivity}

For each SHA (two for each month for this 1990 data set) paired external and internal coefficients can be grouped by Legendre degree and order into values of $(n-m)$ corresponding to the wavelengths around a great circle of longitude. Gauss's (1838) analysis not only allows us to separate the internal from the external parts but the separated terms allows us to treat pairs of corresponding degree and order as separate entities in the field computation. Transfer functions are used to determine a conductivity-depth profile of the subsurface that could be responsible for the observed induced internal fields (see references in introduction section above). For each pair of entities (external and internal) we took only the SHA coefficients having values greater than 0.5 gamma. We followed the earlier studies (Campbell et al., 1994; Arora et al., 1995) that showed only the values with $(n-m)=1$ to be significant because the higher $n-m$ values have been associated with disturbances originating in polar regions. Together with two other computational restrictions (see Arora et al., 1995) we obtained 45 separate conductivity values ranging in depth from 130 to $807 \mathrm{~km}$. Our analysis method depends upon the averaging of values from the distribution of these many individual depth-conductivity determinations. The most likely profile was obtained from the scattered values by using a locally weighted regression fitting described by Cleveland (1979).

In Fig. 9 the asterisks illustrate the conductivity-depth computation results. The line-connected dark circles are the regression fitted values. The analysis method depends on the use of a number of determinations to overcome a variability whose scatter reflects such features as random instrumental drifts, magnetic field contributions produced by other than quiet-time field conditions, and a variability of the source current location. Although the scatter indicates that some inflections in the regression line may be poorly constrained, the trend in the conductivity-depth profile is clearly supported.

The profile starts at $0.025 \mathrm{~S} / \mathrm{m}$ at a depth of $130 \mathrm{~km}$ and rises gradually to about $0.045 \mathrm{~S} / \mathrm{m}$ at $250 \mathrm{~km}$. The profile then steepens to $0.11 \mathrm{~S} / \mathrm{m}$ near $360 \mathrm{~km}$ and rises more gradually to about $0.13 \mathrm{~S} / \mathrm{m}$ at $470 \mathrm{~km}$. There were no further conductivity values until the region of near $800 \mathrm{~km}$ is reached showing about $0.18 \mathrm{~S} / \mathrm{m}$.

\section{Discussion}

A study of the Australian $S q$ for the extremely quiet year, 1965, had been undertaken earlier by Campbell and Schiffmacher (1987, 1988a). In that investigation, boundary stations, PMG and MCQ were also used. However at that time, only data from Gnangara (near Perth) and Toolongi (near Melbourne) were available from the Australian mainland; both were well away from the $S q$ current focus. Therefore, that earlier study was considered to be only a preliminary representation of the Australian quiet-time current system. 


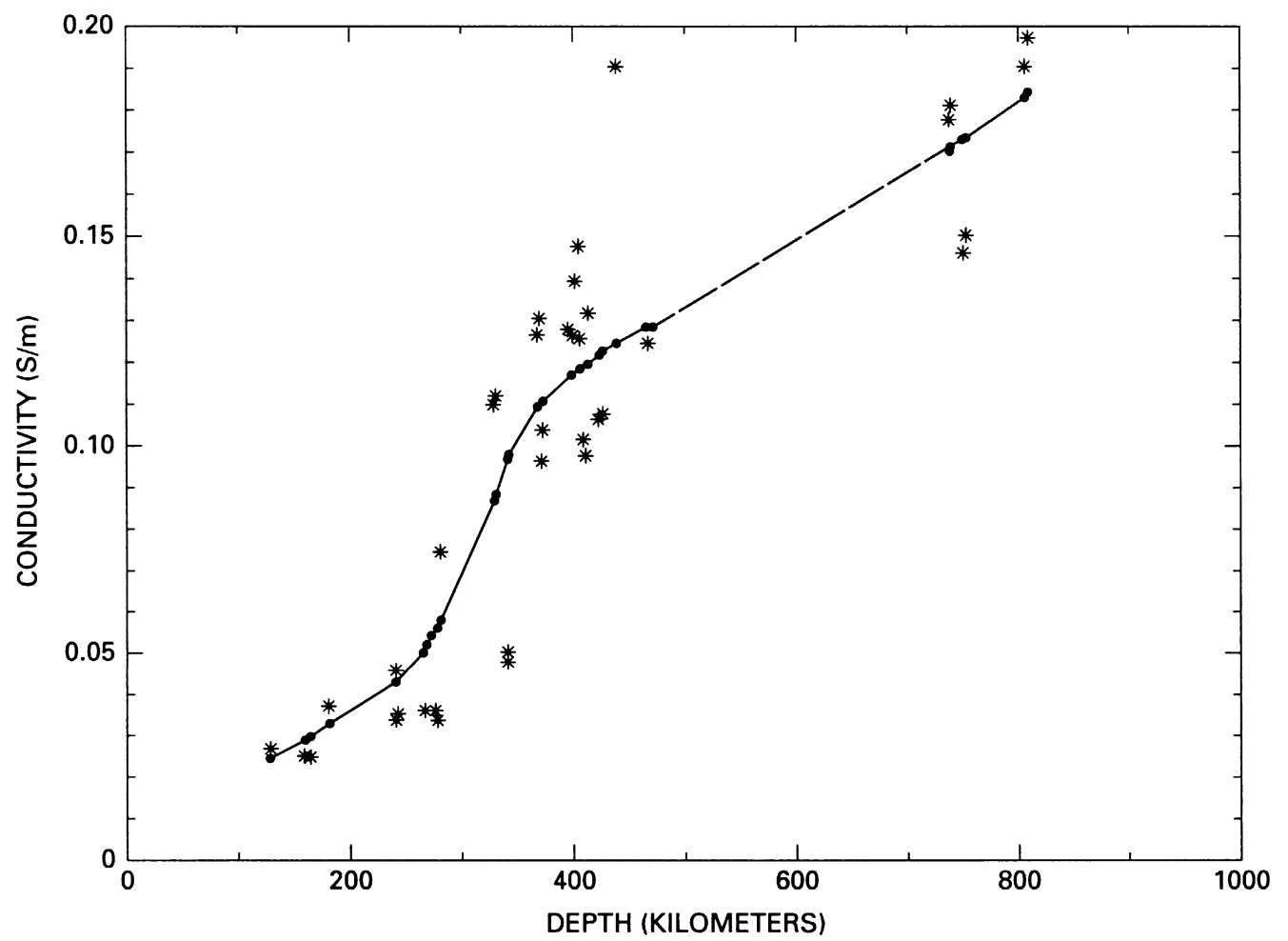

Fig. 9. Electrical conductivity-depth profile of the upper mantle and transition zone based on the Australian Hemisphere model of the quiet daily variation. The solid dots are the locally-weighted regression (Cleveland, 1979) best estimates of the conductivity obtained from the separately computed values (shown as asterisks). Straight lines, solid and dashed, connect the conductivity determinations to emphasize the profile.

The computed yearly track of the external current vortex center appeared in approximately the same locations for 1965 and 1990 with a similar seasonal variation. However, our presently measured summertime and wintertime currents are about 1.5 and 2.4 times as large, respectively, than their 1965 counterparts. This result is consistent with Campbell and Matsushita (1982), who found active year $S q$ currents to be 1.6 and 3.0 times as large, respectively, as the seasonal quiet year currents. The year 1965 remains the quietest on record for international geomagnetic activity, but 1990 was not a particularly quiet year. The change in activity level from an annual sunspot number of 15 in 1965 to 143 in 1990 certainly means that the upper atmosphere ionization and thermospheric wind system (causing the dynamo currents) were greater in 1990 due to greater solar output (see Hoyt and Schatten, 1997). More days of higher Kp index must have been included in the 1990 data sample than in the 1965 analysis.

The earlier mantle conductivity profile of Campbell and Schiffmacher (1987, 1988a) can be compared with our new profile in Fig. 10. The new values are seen to be consistently higher than the old ones by about half-an-order in magnitude. The difference probably reflects the poor mid-latitude continental sampling along the nominal N-S transect in the earlier study, there being only two stations; Toolangi in the extreme southeast of the continent and Gnangara in the extreme southwest (3000 km away), with neither near the locus to the $S q$ focus. Such location could produce larger errors on the earlier estimates of Campbell and Schiffmacher. However, the results do suggest that there may be detectable lateral differences in the electrical conductivity structure of the sub-continental mantle.

It is generally accepted that the mantle is essentially isochemical and that the seismologically-determined boundaries in the mantle are associated with petrological phase changes (Jackson and Rigden, 1997). Whether these boundaries are linked with resolvable changes in electrical conductivity remains unclear, although the present laboratory evidence (Peyronneau and Poirier, 1989; Constable and Duba, 1990; Constable et al., 1992, Poirier and Peyronneau, 1992; Duba and Constable, 1993) favors a smooth temperature-controlled variation of conductivity through the mantle (noted by Heinson and Constable, 1992). In particular, laboratory measurements to date suggest that there are no major transitions in conductivity in the mantle at $400 \mathrm{~km}$. Nevertheless, the shape of Fig. 9 does suggest some mantle zonation, so it is worthwhile comparing our model with the inferred seismological and petrological boundaries.

The upper mantle (at depths shallower than about $350 \mathrm{~km}$ ) exhibits distinct lateral heterogeneity with lowest seismic velocities below mid-ocean ridge systems, and highest velocities beneath old continental blocks extending to depth of 250 to $300 \mathrm{~km}$. This lateral variability in seismic velocities is believed to be primarily thermal rather than compositional in origin because of the weak velocity-composition dependence in the ultramafic lithologies that dominate the upper mantle (Jackson and Rigden, 1997).

The major mantle discontinuities occur in the transition zone $(350$ to $750 \mathrm{~km}$ ) and are attributed to two pressureinduced phase changes as peridotic upper mantle minerals (60\% olivine by volume, pyroxenes, garnet, and minor constituents), with crystal structures based on $\mathrm{SiO}_{4}$ tetrahedra, 
are progressively replaced by denser, stiffer structures having octahedrally-coordinated silicon. Jackson and Ridgen (1997) concluded that the phase changes of olivine to wadsleyite and, subsequently, the disproportionation of ringwoodite to yield $(\mathrm{Mg}, \mathrm{Fe}) \mathrm{SiO}_{3}$ perovskite and magnesiowustite occur within very restricted pressure ranges that are consistent with depths and sharpness of the 410-km and $660-\mathrm{km}$ discontinuities that are observed world-wide (e.g., Shearer, 1991). Minor discontinuities have been reported at greater depths. In the Australian region, the 410$\mathrm{km}$ and $660-\mathrm{km}$ boundaries are clearly evident, but a $520-$ $\mathrm{km}$ seismic discontinuity is not generally identified. The base of the lithosphere is marked by a boundary at $210 \mathrm{~km}$ (cf. Kennett et al., 1994). This feature is not clearly resolved throughout the region and is attributed to fluctuations in seismic anisotropy rather than to chemical composition or phase changes (Kennet, personal communication).

The boundaries discussed above are marked on Fig. 10. Our conductivity profile does not resolve a discontinuity at the base of the lithosphere at $210 \mathrm{~km}$, but starts to rise at about $250 \mathrm{~km}$. The conductivity increases most rapidly with depth through the upper mantle to its base at a depth of about $350 \mathrm{~km}$. This behavior does not match a specific seismic discontinuity, but does correspond to the maximum depths at which the roots of old continent are detectable in seismic data. We have no conductivity estimates between 470 and $730 \mathrm{~km}$, but any substantial variation in conductivity through this region seems to be ruled out because the regression fit changes by less than $0.05 \mathrm{~S} / \mathrm{m}$ over this interval.

Figure 10 also shows a selection of global-average conductivity profiles for the mantle. The models of Bott (1982) and Stacey (1992) are notional curves based on a synthesis of many individual results. Our estimates for the lower mantle conductivity appear to be consistently lower by almost an order of magnitude, than the global ones. In the upper mantle our estimates are higher, again by about an order of magnitude, than the global estimates, with the exception of Parker's (1971) Backus-Gilbert inversion result. These systematic differences are quite big, even given the large uncertainties in the mantle conductivity estimates, and suggest that either the earlier determinations are in error or that major regional variations should be considered.

For reasons mentioned earlier, our analysis uses data from the equator to the pole, but is weighted towards data from latitudes near the $S q$ focus. Consequently, should there be any detectable lateral variation in mantle conductivity, we would expect our conductivity values to be a better match to regional profiles from central Australia than to global averages. A comparison with profiles obtained for the Australian region by geomagnetic depth sounding (GDS) methods is shown in Fig. 11.

Lilley et al. (1981; we will call this reference LWS) found "a substantial difference in conductivity structure between central and southeast Australia. Beneath central Australia,

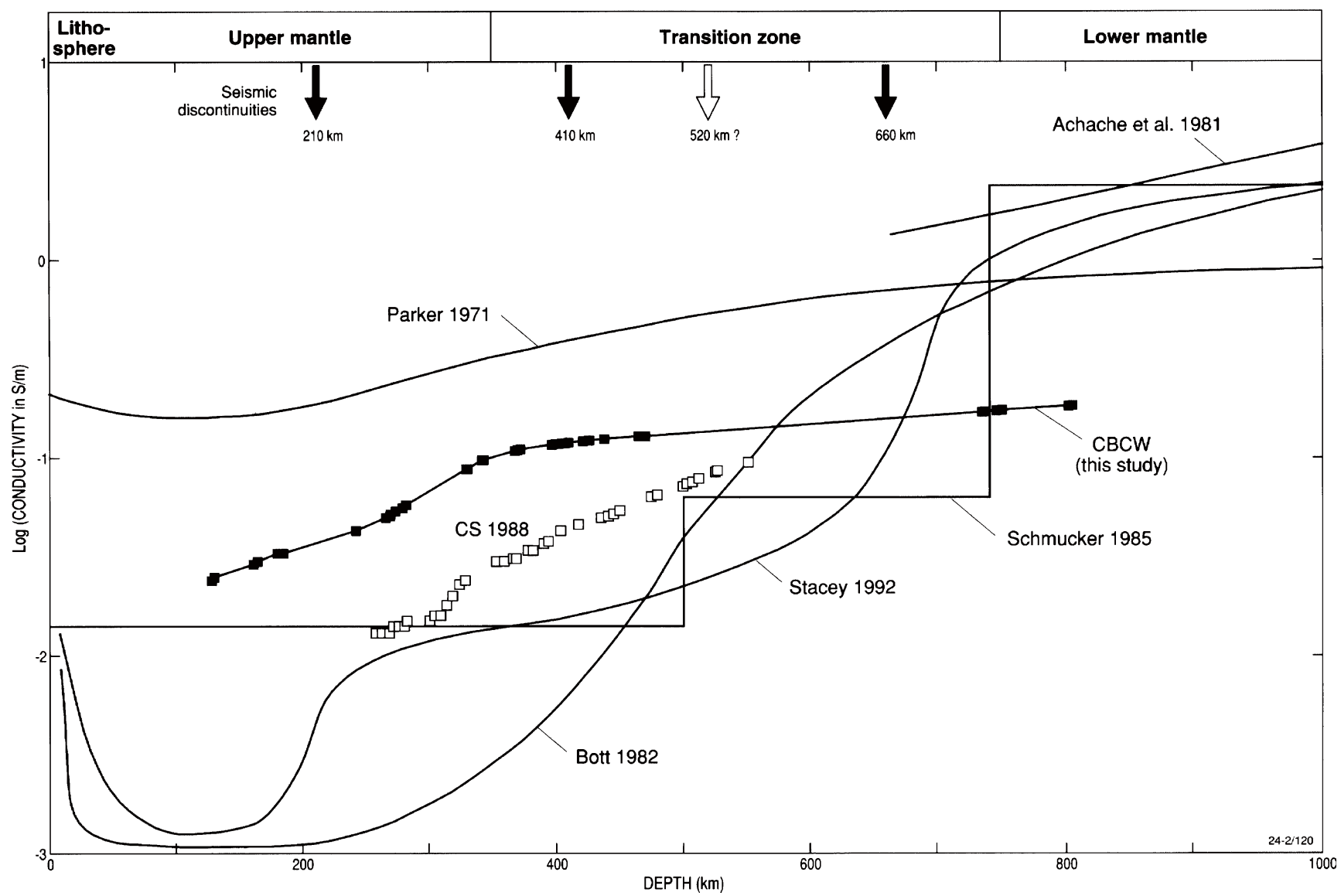

Fig. 10. Comparison of the Australian hemisphere conductivity profiles with global models. The new result for the Australian hemisphere is labeled CBCW (solid squares); CS 1988 (open squares) is the earlier Australian hemisphere result of Campbell and Schiffmacher (1988). The various global models are labelled according to their bibliographic references. 
the structure is consistent with a traditional continental geotherm and published laboratory measurements on the temperature dependence of the electrical conductivity of recognized upper-mantle crystalline olivine materials". Note that eastern Australia is geologically younger and geophysically distinct from central Australia. In Fig. 11, the shaded zone shows the range of models designated by LWS to be acceptable fits to GDS data for central Australia. Our conductivity profile, shown as a bold line with solid squares in Fig. 11, lies substantially within this range, falling outside it only at depths greater than $550 \mathrm{~km}$ (where all models have poor resolution). By contrast our regression profile is clearly a poor fit to LWS's range of acceptable models for eastern Australia (hatched region in Fig. 11).

Takeda (1991) has studied electric currents induced in the ocean by $S q$ fields and believes that our conductivity measurements may be affected by the ocean. We do not consider his oceanic work as applicable to our continental results. Our analysis is naturally weighted toward the region of the
$S q$ current focus, which is located midcontinent for this Australian region. In addition, contrary to that author's statement that our method assumes "the induced currents flow only in the mantle", our computations do not assume a depth to the conductivity but rather let the relationships of the observed external and internal fields determine the profiles.

No GDS studies have yet been completed in the equatorial sector of our longitudinal transect. Likewise, we have no information for the Southern Ocean (although F. E. M. Lilley is currently undertaking one such study). However, White and Heinson (1994) have followed earlier GDS work by White and Polatayko $(1978,1985)$ to obtain an electrical conductivity profile for the continental-ocean boundary of South Australia (using a transect of stations going from the land to just beyond the continental shelf south of station PTA in our Fig. 1). Their profile is marked as a short-dashed line in Fig. 11 with uncertainty estimates (Heinson, personal communication) shown by the dotted zone. Our profile

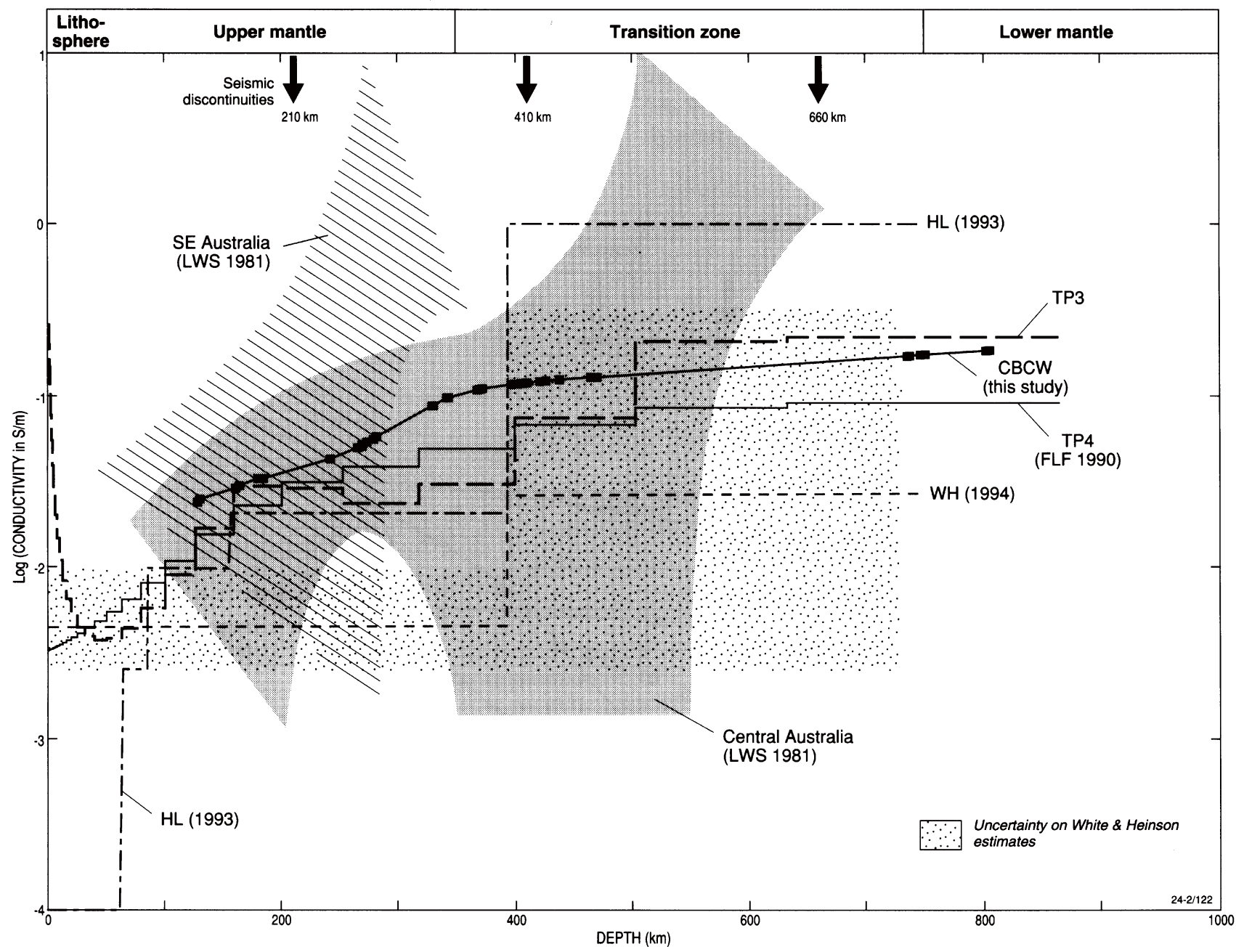

Fig. 11. Comparison of our Australian Hemisphere profile (squares and bold line) with regional electrical conductivity models. The shaded zone shows the range of acceptable models that fit magnetometer array data for central Australia (Lilley et al., 1981); the hatched zone shows the range of models that fit array data for southeastern Australia (by the same authors). Models for the middle of the Tasman Sea (Ferguson, Lilley, and Filloux, 1991) are shown by the bold stepped dashed line (site TP3) and the stepped thin line (site TP4). WH(1994) is the result of White and Heinson (1994) that was based on a transect of the GDS stations across the continent-ocean boundary of South Australia. HL(1993) is the profile obtained by Heinson and Lilley (1993) from a re-analysis of the Tasman sea data. Below about $400 \mathrm{~km}$, all the models lack resolution. Observe the uncertainty in WH(1994), shown stippled; HL(1993) could have been placed at $0.1 \mathrm{~S} / \mathrm{m}$ without affecting the fit to the data. 
indicates a conductivity about an order of magnitude greater than White and Heinson's in the Upper Mantle, but better agreement (given the very large uncertainties) in the Transition Zone (i.e. below about $400 \mathrm{~km}$ ).

We may also compare our results with profiles obtained by Ferguson et al. (1991) for the middle of the Tasman Sea (sites TP3 and TP4 between the Australian mainland and Lord Howe Rise). Ferguson et al.' s conductivity profiles are shown in Fig. 11 by the long-dashed and continuous lines, respectively. There is good general agreement with our new profile; the Tasman profiles having lower conductivities than our model above $400 \mathrm{~km}$, and similar values below. Heinson and Lilley (1993) have applied a more sophisticated analysis of the Tasman Sea data incorporating thin-sheet modelling of the ocean layer and a technique to remove 3D distortion from the observed seafloor MT data. Heinson and Lilley's profile is shown as a dash-dot line in Fig. 11. They required a conductivity of less than $10^{-4} \mathrm{~S} / \mathrm{m}$ for the lithosphere in order to fit the observed anisotropy in seafloor MT data, but apart from this feature, Heinson and Lilley's profile is similar to those of Ferguson et al. (1991) for TP3 and TP4. All the profiles lie within LWS's range of acceptable models for central Australia.

The comparisons we make with models at depths greater than $400 \mathrm{~km}$ must be treated with caution because all the models lack resolution at such depths. The scale of this uncertainty is illustrated on Fig. 11 graph of White and Heinson's (1994) model (shown stippled); changes in conductivity of two orders of magnitude can be made without affecting their data fit.

\section{Conclusions}

A new model of the $S q$ current system for the Australian "hemisphere" for 1990 has been obtained from magnetometer data using a chain of geomagnetic records going from the equator, through central Australia, to the pole. Summertime and wintertime ionospheric currents in 1990 had focus values of 200 and $80 \mathrm{kA}$ and were 1.5 to 2.4 times greater, respectively, than they were in the solar-quiet year of 1965 . The yearly track of the $S q$ current focus that passes across central Australia has a seasonal position and movement that were similar in 1965 and 1990.

The mantle electrical conductivity profile obtained from the $S q$ model is dominated by data from the central Australian mainland. The principal features of the new profile are (1) the conductivity gradient thought the lower part of the upper mantle is steeper than in the transition zone, (2) the base of the Australian lithosphere and seismic discontinuities at $410 \mathrm{~km}$ and $660 \mathrm{~km}$ are not evident in the profile, (3) conductivities are higher than average global models for the upper mantle, broadly similar in the transition zone, and lower in the lower mantle, (4) the profile lies within the range of models determined from earlier array studies in central Australia for the upper mantle and into the transition zone, and (5) at greater depths, below about $400 \mathrm{~km}$, notwithstanding the poor resolutions a such depths, the profile is in agreement with the values obtained from previous electromagnetic studies carried out across the continentocean boundary of South Australia and on the floor of the Tasman Sea.
Acknowledgments. The first author, WHC, was working for the Branch of Global Seismology and Geomagnetism of the U.S. Geological Survey, on assignment in Australia, during the period of data processing for this study. He wishes to thank that organization for the USGS travel grant and the corresponding visiting scientist grants provided by AGSO and Flinders University of South Australia that made possible this cooperative study. The authors also thank the Geological Survey Division, Papua New Guinea for providing data from Port Moresby Observatory, Stewart Dennis for preparing data from Macquarie Island Observatory, Phil McFadden for producing Fig. 1, and Ted Lilley, Anton Hales, Ian Jackson, and Brian Kennett for valuable discussions. Comments and information provided by Graham Heinson were particularly helpful. CEB and WW publish with permission from the Executive Director, Australian Geological Survey Organisation.

\section{References}

Arora, B. R., W. H. Campbell, and E. R. Schiffmacher, Upper mantle electrical conductivity in the Himalayan region, J. Geomag. Geoelectr., 47, 653-665, 1995.

Bott, M. H. P., The Interior of the Earth: Its Structure, Constitution, and Evolution, 403 pp., Edward Arnold Company, London, 1982.

Campbell, W. H., The upper mantle conductivity analysis method using observatory records of the geomagnetic field, Pure and Appl. Geophys., 125, 427-457, 1987.

Campbell, W. H., Differences in geomagnetic $S q$ field representations due to variations in spherical harmonic analysis techniques, J. Geophys. Res., 95, 20,923-20,936, 1990.

Campbell, W. H., Introduction to Geomagnetic Fields, 304 pp., Cambridge University Press, New York, 1997.

Campbell, W. H. and R. S. Anderssen, Conductivity of the subcontinental upper mantle: an analysis using quiet-day records of North America, $J$. Geomag. Geoelectr., 35, 367-382, 1983.

Campbell, W. H. and S. Matsushita, $S q$ Currents: A comparison of quiet and active year behavior, J. Geophys. Res., 87, 5305-5308, 1982.

Campbell, W. H. and E. R. Schiffmacher, Quiet ionospheric currents of the Northern Hemisphere derived from geomagnetic field records, $J$. Geophys. Res., 90, 6475-6486, 1986.

Campbell, W. H. and E. R. Schiffmacher, Quiet ionospheric currents and Earth conductivity profile computed from quiet-time geomagnetic field changes in the region of Australia, Aust. J. Phys., 40, 73-87, 1987.

Campbell, W. H. and E. R. Schiffmacher, Quiet ionospheric currents of the Southern Hemisphere derived from geomagnetic records, $J$. Geophys. Res., 93, 933-944, 1988a.

Campbell, W. H. and E. R. Schiffmacher, Upper mantle electrical conductivity for seven subcontinental regions of the Earth, J. Geomag. Geoelectr., 40, 1387-1406, 1988b.

Campbell, W. H., E. R. Schiffmacher, and H. W. Kroehl, Global quiet day field variation model WDCA-SQ1, EOS, Trans. Amer. Geophys. Un., 70, 66 and 74, 1989.

Campbell, W. H., E. R. Schiffmacher, and B. R. Arora, Quiet geomagnetic field representation for all days and latitudes, J. Geomag. Geoelectr., 44, 459-480, 1992.

Campbell, W. H., B. R. Arora, and E. R. Schiffmacher, External $S q$ currents in the India-Siberia region, J. Geophys. Res., 98, 3741-3752, 1993.

Campbell, W. H., B. R. Arora, and E. R. Schiffmacher, Polar cap field response to IMF $B_{y}$ sector changes on quiet days at a longitude line of observatories, J. Geomag. Geoelectr., 46, 735-746, 1994.

Chamalaun, F. H. and C. E. Barton, The large-scale conductivity structure of Australia, J. Geomag. Geoelectr., 45, 1209-1212, 1993 a.

Chamalaun, F. H. and C. E. Barton, Electromagnetic induction in the Australian crust: results from the Australia-wide Array of Geomagnetic Stations, Explor. Geophys., 24, 179-186, 1993b.

Chamalaun, F. H. and R. A. Walker, A microprocessor based digital fluxgate magnetometer for geomagnetic deep sounding studies, $J$. Geomag. Geoelectr., 34, 491-507, 1982.

Chapman, S., The solar and lunar diurnal variation of the Earth's magnetism, Phil. Trans. Roy. Soc. London, A218, 1-118, 1919.

Chapman, S. and J. Bartels, Geomagnetism, 1049 pp., Oxford University 
Press, Oxford, 1940.

Cleveland, W. S., Robust locally weighted regression and smoothing scatter plots, J. Amer. Statistical Assn., 74, 828-833, 1979.

Constable, S. and A. Duba, Electrical conductivity of olivine, a dunite, and the mantle, J. Geophys. Res., 95, 6967-6978, 1990.

Constable, S., T. J. Shankland, and A. Duba, The electrical conductivity of an isotropic olivine mantle, J. Geophys. Res., 97, 3397-3403, 1992

Duba, A. and S. Constable, The electrical conductivity of Lherzolite, $J$. Geophys. Res., 98, 11885-11899, 1993.

Ferguson, I. J., F. E. M. Lilley, and J. H. Filloux. Geomagnetic induction in the Tasman Sea and electrical conductivity structure beneath the Tasman seafloor, Geophys. J. Int., 102, 299-312, 1991.

Gauss, C. F., Allgemeine Theorie des Erdmagnetismus, in Resultate aus den Beobachtungen des magnetischen Vereins im Yahr 1838, edited by C. F. Gauss and W. Weber, translated from the German by E. Sabine and R. Taylor, Sci. Mem. Select. Trans. Foreign Acad. Learned Soc. Foreign J., 2, 184-251, 1841.

Heinson, G. S. and S. Constable, The electrical conductivity of the oceanic upper mantle, Geophys. J. Int., 110, 159-179, 1992.

Heinson, G. S. and F. E. M. Lilley, An application of electromagnetic thin-sheet modelling to the Tasman Sea, Phys. Earth Planet. Int., 81, 231-251, 1993

Hoyt, D. V. and K. H. Schatten, The Role of the Sun in Climate Change, 279 pp., Oxford Press, Oxford, 1997.

Jackson, I. and S. Rigden, Composition and temperature of the Earth's mantle: seismological models interpreted through experimental studies of mantle materials, in The Earth's Mantle: Composition, Structure, and Evolution, edited by I. Jackson, Cambridge Univ. Press, 1997 (in press).

Kennett, B. L. N., O. Gudmundsson, and C. Tong, The upper-mantle $S$ and $\mathrm{P}$ velocity structure beneath northern Australia from broad-band observations, Phys. Earth Planet. Int., 86, 85-98, 1994.

Lilley, F. E. M., The analysis of daily variations recorded by magnetometer arrays, Geophys. J. Roy. Astr. Soc., 43, 1-16, 1975.

Lilley, F. E. M., D. V. Woods, and M. M. Sloane, Electrical conductivity profiles and implications for the absence or presence of partial melting beneath central and south east Australia, Phys. Earth Planet. Int., 25 419-428, 1981.

Matsushita, S. and H. Maeda, On the geomagnetic quiet solar daily variation field during the IGY, J. Geophys. Res., 70, 2535-2558, 1965.

Maxwell, J. C., Treatise on Electricity and Magnetism, 566 pp., Cambridge University Press, Cambridge, 1873.
Parker, R. L., The inverse problem of electrical conductivity in the mantle, Geophys. J. Roy. Astr. Soc., 22, 121-138, 1971.

Parkinson, W. D., An analysis of the geomagnetic diurnal variation during the International Geophysical Year, BMR Bulletin No 173, Australian Geological Survey Organisation, Canberra, 196 pp., 1977.

Peyronneau, J. and J.-P. Poirier, Electrical conductivity of the Earth's lower mantle, Nature, 343, 537-539, 1989.

Poirier, J.-P. and J. Peyronneau, Experimental determination of the electrical conductivity of the material of the Earth's lower mantle, in High Pressure Research: Application to Earth and Planetary Sciences, Geophysics Monograph No. 67, edited by Y. Syono and M. H. Manghani, pp. 77-87, American Geophysical Union, Washington, D.C., 1992.

Schmucker, U., An introduction to induction anomalies, J. Geomag. Geoelectr., 22, 9-33, 1970.

Schuster, A., The diurnal variation of terrestrial magnetism, Philos. Trans. Roy. Soc. Lon., Ser. A, 180, 467-518, 1889.

Schuster, A., The diurnal variation of terrestrial magnetism, Philos. Trans. Roy. Soc. Lon., Ser. A, 208, 163-204, 1908.

Shearer, P. M., Constraints on upper mantle discontinuities from observations of long-period reflected and converted phases, J. Geophys. Res., 96, 18147-18182, 1991

Stacey, F. D., Physics of the Earth, 3rd edition, 513 pp., Brookfield Press, Brisbane, 1992.

Stening, R. J. and P. A. Hopgood, Geomagnetic quiet daily variations in the Australian region-information from a new station in Charters Towers, J. Atmos. Terr. Phys., 53, 959-964, 1991.

Takeda, M., Electric currents in the ocean induced by the geomagnetic $S q$ field and their effects on the estimation of mantle conductivity, Geophys. J. Int., 104, 381-385, 1991.

White, A. and G. Heinson, Two-dimensional conductivity structure across the southern coastline of Australia, J. Geomag. Geoelectr., 46, 1067-1081, 1994.

White, A. and O. W. Polatayko, The coast effect in geomagnetic variations in South Australia, J. Geomag. Geoelectr., 30, 109-120, 1978.

White, A. and O. W. Polatayko, Electrical conductivity anomalies and their relationship with tectonics of South Australia, Geophys. J. Roy. Astr. Soc., 80, 757-771, 1985.

W. H. Campbell (e-mail: whc@ngdc.noaa.gov), C. E. Barton, F. H. Chamalaun, and W. Welsh 